\title{
Big data y redes sociales: un acercamiento al mercado de trabajo sumergido de Navarra en los medios sociales y en la prensa escrita
}

\section{Antidio Martínez de Lizarrondo Artola}

Observatorio de la Realidad Social, Gobierno de Navarra

amartart@navarra.es

\section{Laura Pérez Villanueva}

Observatorio de la Realidad Social, Gobierno de Navarra Iperezvi@navarra.es

\begin{abstract}
Artikulu honek Nafarroko ekonomia ezkutuaren tratamendu mediatiko eta onlinekoaren hurbilketa berri bat aurkezten du, big data tekniken bitartez. Hitz gako jakin batzuetatik abiatuta, 2016-2018 urteetako ezkutuko lan merkatuaren egoera ikertu da bi tresna erabiliaz (web mining interneten sortutako informazioaren kasuan eta MyNews idatzitako prentsarentzat). Kontzeptu honek izaera polisemikoa izan arren, nabarmentzekoa da aztertutako erreferentzien parte handi bat zerga iruzurrari buruz jarduten dela, eta ikuspuntu kritiko batetik egiten dela gainera, fenomeno honekiko existitzen den onarpen mailaz haratago. Halaber, sare sozialetan gai honen inguruko komentarioak ez dira apenas agertzen eta ez da eratzen elkarrizketarako kanal gisa. Honek espazio interesgarria irekitzen du administrazioek sare sozialetan presentzia handiagoa izan dezaten sentsibilizazio neurriak abiaraziz eta azpijoko honen aurrean eraginkortasun handiagoa lortuz.
\end{abstract}

\section{GAKO-HITZAK:}

Lurpeko ekonomia, zerga iruzurra, sare sozialak, prentsa idatzia, big data.
Este artículo presenta una aproximación al tratamiento mediático y online de la economía sumergida en Navarra realizada mediante técnicas de big data. En base a determinadas palabras clave y a través de dos instrumentos (web mining para la información generada en Internet y MyNews para la prensa escrita) se ha realizado un estudio acerca del mercado de trabajo sumergido en el periodo 2016-2018. Pese al carácter polisémico de este concepto, gran parte del contenido de las referencias analizadas versa sobre el fraude fiscal y presenta connotaciones críticas a pesar de la condescendencia hacia este fenómeno. Asimismo, la presencia en redes sociales de comentarios sobre esta temática es prácticamente inexistente y no se configura como canal de conversación. Esto abre un interesante espacio para que las administraciones adquieran mayor presencia en las redes sociales poniendo en marcha medidas de concienciación y mostrando mayor eficacia contra esta lacra.

\section{Palabras Clave:}

Economía sumergida, fraude fiscal, redes sociales, prensa escrita, big data. 


\section{Introducción}

El ámbito de estudios sobre los encuadres de las redes sociales y de los medios de comunicación y su importancia a la hora de establecer la agenda política ha tenido un recorrido y desarrollo muy importante durante las últimas décadas, especialmente en lo que va de siglo. Ahora bien, dicho ámbito de estudios sociales se ha centrado principalmente en el examen de la relevancia de la ideología de los medios de comunicación y el tratamiento mediático de los diversos fenómenos sociales filtrados a través de ese prisma ideológico. No parece que la economía sumergida sea un tema polémico para hallar interpretaciones mediadas por la ideología, pero sí hay estudios que han analizado diversos aspectos 0 actividades que se integran en este concepto de economía sumergida o sobre colectivos especialmente afectados en su definición.

Este artículo recoge una doble aproximación al tratamiento mediático y online de la economía sumergida en la Comunidad Foral de Navarra. Las particularidades más novedosas de esta investigación se deben a la metodología empleada. A través de la utilización de dos instrumentos (el web mining ${ }^{1}$ como disciplina de rastreo, búsqueda y análisis de la información generada en Internet, y My News ${ }^{2}$ para la consulta a la hemeroteca de los principales medios de comunicación escritos de Navarra), se ha realizado un estudio acerca del mercado de trabajo sumergido o informal en Navarra.

En base a determinadas palabras clave, el análisis abarca el periodo 2016-2018 tanto para la selección de noticias de prensa escrita como para la búsqueda de referencias en los medios sociales en Internet. A partir de ahí se muestra un análisis descriptivo y se estudia el encuadre que realizan las redes sociales y la prensa acerca de las temáticas relacionadas con la economía sumergida, todo ello con el fin de conocer ciertas particularidades sobre la situación, la percepción e imagen que se ofrecen sobre un fenómeno difícil de definir y cuantificar.

Junto al análisis de las noticias mediatizadas por la ideología, destaca la importancia que tienen la frecuencia y el sentido de dichas noticias en los medios y en las redes sociales. De esta manera, además de que el prisma ideológico afecta a la interpretación que los lectores hacen sobre un tema en particular, los medios de comunicación y las redes sociales pueden fijar el carácter manifiesto o latente de las problemáticas. En este caso concreto y pese al carácter polisémico del concepto de "economía sumergida", de entre todas las referencias

${ }^{1}$ La empresa Quor tuvo el encargo por parte del Observatorio de la Realidad Social de analizar las redes sociales mediante esta técnica, de forma que aportó los datos necesarios para el estudio del Observatorio.

${ }^{2}$ El SIIS-Fundación Eguía-Careaga se encargó de hacer las gestiones para la utilización de My News y quien analizó los datos resultantes para que el Observatorio de la Realidad Social completara la investigación. analizadas en prensa escrita destaca que gran parte de su contenido versa sobre el fraude fiscal generado por el mercado de trabajo sumergido, con unas connotaciones más críticas a pesar de la condescendencia social hacia este fenómeno. Además, es muy curioso que la presencia en redes sociales de comentarios sobre esta temática sea prácticamente inexistente y no se presenta como un canal de conversación con usuarios internautas opinantes.

\section{Objetivos de la Investigación}

Los objetivos generales de la doble investigación han sido los siguientes:

- Medir la visibilidad y presencia online y en la prensa navarra de la economía sumergida y sus diversas actividades.

- Categorizar y evaluar el clima de opinión mediático y online sobre el mercado de trabajo sumergido.

- Identificar, etiquetar y describir las temáticas sobre las que se habla, estableciendo y analizando la posición valorativa al respecto.

Con todo ello, los objetivos específicos han tratado de:

- valorar qué se comenta a nivel de redes sociales y en prensa escrita sobre el mercado de trabajo sumergido;

- averiguar qué nivel de aceptabilidad o rechazo tiene la economía sumergida en las redes sociales y en la prensa escrita;

- determinar qué valoración social hay del control e inspección acerca del mercado de trabajo sumergido;

- concretar en qué sectores se manifiesta que hay más trabajo sumergido;

- identificar qué condiciones laborales van ligadas a él; e

- investigar qué tipos de perfil de personas se encuentran en esta práctica.

\section{Base teórica y antecedentes metodológicos}

Maxwell E. McCombs y Donald L. Shaw presentaron en 1972 un estudio fundacional sobre la influencia de los medios de masas y su importancia en el proceso selectivo y en la contextualización de la información (McCombs y Shaw, 1972). La teoría subraya la función de la prensa en el establecimiento o fijación de la agenda política ("agenda-setting”) y se ve confirmada en su investigación sobre la dirección del voto por parte de los habitantes de Chapel Hill (Carolina del Norte) y la relevancia de los mensajes mediáticos en las elecciones presidenciales de 
Estados Unidos en 1968. Afirman que los medios de comunicación fuerzan a dirigir la atención a ciertos temas: construyen y proporcionan imágenes constantemente, con las que los individuos que conforman la masa reciben sugerencias sobre cómo pensar, saber y hasta llegar a sentir.

McCombs y Shaw citan en ese artículo a otro colega, Bernard C. Cohen, que en 1963 explicita la función de establecimiento de la agenda que cumple la prensa: "Puede que no haya sido exitosa a la hora de decir a la gente qué es lo que tiene que pensar, pero sí ha tenido un increíble éxito al transmitir a sus lectores en qué tienen que pensar" (McCombs y Shaw 1972: 177). Su hipótesis principal afirma que los medios de masas establecen la agenda de cada campaña política e influencian la emergencia de las actitudes de la opinión pública hacia los diversos temas políticos. Parece lógico que los atributos de un tema afecten a su relevancia mediática, pero en un segundo nivel, la relevancia temática de los medios de comunicación influye sobre las actitudes y opiniones.

Tal y como explican Carlos Muñiz y Juan José Igartua en el artículo "Encuadres noticiosos e inmigración. Un análisis de contenido de la prensa y televisión españolas" (Muñiz e Igartua, 2004), en el caso de las personas que han participado en procesos migratorios se presentan posturas reduccionistas que son contrastables en datos, por ejemplo, cuando se exageran los porcentajes al cuantificar el número de personas migrantes o se extienden mensajes xenófobos y rumores. En ese aspecto por el que se atribuyen características a los sujetos o colectivos, la teoría de estos autores enlaza en el segundo nivel de la función de los medios de comunicación de la fijación de la agenda con el "frame analysis" o anáisis de marcos de Erving Goffman (2006), por la influencia que tienen sobre cómo pensar sobre estos asuntos. El análisis de marcos va más allá al afirmar que los encuadres de los medios de comunicación son una representación de la realidad social construida (o contextualizada) por la persona que redacta la noticia.

Canel et al. (1996) llevaron a cabo un estudio pionero en el que analizaron los tipos de agenda para valorar el efecto de los medios en la opinión pública sobre las variables de educación, consumo de medios, sexo e ideología en el contenido de 136 noticias de prensa y 36 de televisión. Este estudio confirmaba la hipótesis de la función de fijación de la agenda por parte de los medios, una mayor eficacia por parte de la prensa escrita que la televisión en su función de establecimiento de la agenda y una menor vulnerabilidad de las personas activas interesadas en política.

En este sentido, cabe destacar otros dos estudios muy diferentes entre sí que analizan el rol que juegan los medios de la prensa escrita. En primer lugar, Canel (1999) analiza en un artículo políticas de opinión, editoriales, portadas, redacción de titulares y la información sobre el Gobierno de $E l$
País, El Mundo y $A B C$. Por otra parte, Igartua et al. (2005) llevaron a cabo un análisis de 819 noticias de El País, El Mundo, ABC y La Razón relacionadas con la inmigración. En ambos casos se observa su relevancia en la formación y modulación de la opinión social; así, se demostraba que los encuadres noticiosos provocan efectos cognitivos reseñables y que el énfasis mediático de determinados atributos de los objetos sociales puede condicionar la percepción sobre ellos.

Ligado a la influencia de los mass media tradicionales, es obvia la exponencial incidencia que están teniendo las redes sociales en nuestras vidas. Del Fresno (2011: 30) refiere que "la web social es el fenómeno socio-tecnológico más significativo de las últimas décadas con un impacto que está lejos de poder darse por terminado [...], lo que afecta a la forma en que nos comprendemos de forma individual y colectiva, marcando una clara línea de separación para el conjunto de la población entre un antes y después de la aparición de Internet”.

Hoy en día creamos estructuras virtuales en torno a la Red y sus múltiples derivaciones, por lo que el análisis de redes sociales se convierte en una herramienta cada vez más útil para comprobar las causas y efectos de determinados fenómenos sociales. En este sentido, Sanz (2003) señala que la idea fundamental de este análisis es que las interacciones entre individuos en la red social tienen un impacto relevante en el comportamiento de los actores, así como resultados en las estructuras de poder y en los procesos de aprendizaje. Dicho de otro modo, el análisis de redes analiza cómo la estructura social de relaciones afecta a las ideas y a la conducta de las personas, y las redes sociales son a la vez causa y resultado de las conductas de los individuos. Por ello cada vez es más frecuente que las empresas se preocupen por su reputación de marca online, de forma que se emplean técnicas que identifican, extraen, clasifican y analizan las opiniones que los usuarios diseminan en las redes sociales sobre diferentes marcas, productos y servicios. La novedad en este caso es la aplicación de esta técnica que examina la "reputación online" (Del Fresno 2011), para analizar los discursos y opiniones que sobre una determinada temática social expresan los sujetos en la Web.

\subsection{Qué se entiende por economía sumergida en España}

Si nos centramos en la conceptualización de nuestro objeto de estudio, en el análisis La Economía Sumergida en Navarra (Mauleón y Sardá, 2014) se advierte de las dificultades a la hora de determinar qué es la economía sumergida y sobre sus múltiples definiciones. Por su parte, el Consejo Económico y Social (CES) de España refiere que reserva:

[...] el concepto de economía sumergida para el conjunto de actividades de producción de bienes y prestación de servicios para el mercado 
que eluden normas, tanto fiscales - en sentido amplio-como de cualquier otro tipo con contenido económico, entre las que se encuentran las regulaciones laborales, pero también otras, como las referidas al medio ambiente, las normas técnicas, las de seguridad, etc." (Consejo Económico y Social, 1999: 11)

Asimismo, es preciso tener en cuenta que el CES español define la "economía informal” como el conjunto de actividades producidas en el hogar que:

[...] han de quedar al margen de la consideración de la economía sumergida pues, aunque claramente generan valor, éste no es mensurable con los mismos parámetros que la producción del mercado, ni le serían de aplicación (salvo casos tasados) las regulaciones que, con carácter general, se establecen para la producción de mercado. (CES 1999: 8)

Mauleón y Sardá (2014: 6) toman en consideración la definición de la OCDE, en la que "la economía sumergida consiste en actividades que son productivas en sentido económico y relativamente legal (si ciertos estándares o regulaciones se cumplen), pero que son escondidas deliberadamente a las Administraciones Públicas". En cambio, en un informe de la Fundación de las Cajas de Ahorros (Vaquero et al., 2015: 4), la economía sumergida se dice que "engloba las actividades económicas productivas ilegales (tráfico de drogas, tráfico de personas, blanqueo de capitales, etcétera) pero también la “economía irregular”. Esto es, operaciones que permanecen ocultas a la Administración, que tratan de evitar su fiscalización, eluden el pago de las cuotas a la Seguridad Social e incumplen las normas laborales o administrativas".

\subsection{Una aproximación a la incidencia de la economía sumergida en Navarra}

Mauleón y Sardá (2014) publicaron un novedoso análisis sobre la evolución de la economía sumergida en el periodo 1990-2012 en Navarra, que es el estudio más reciente en esta comunidad autónoma sobre esta temática; por ello es preciso referir en este epígrafe sus principales conclusiones. Estos autores identifican la tasa de paro como el factor fundamental que determina el peso de la economía sumergida, al tiempo que también consideran apreciable la incidencia que en su aumento tiene el incremento de la imposición directa. Para el año 2012 calculaban en España un valor en torno al $23 \%$ de la economía sumergida sobre la economía contabilizada (el PIB). en el sector servicios, seguido de la construcción y, en último lugar, industria y agricultura.

Este trabajo estima que en ese periodo el peso de la economía sumergida en Navarra osciló entre el $13 \%$ y el $18 \%$ del PIB, es decir, en casi todos los años se situó hasta cinco puntos por debajo de la media estatal. Comparativamente, en 2012 Navarra era con el $18 \%$ la segunda comunidad autónoma donde menos peso tenía la economía sumergida, tras Madrid (16,2\%). En el otro extremo se encontraban Extremadura y Andalucía (29,1\% y $27,3 \%$, respectivamente). Las causas de esta menor incidencia en Navarra hay que buscarlas en las características económicas de la comunidad foral, donde sectores en los que tradicionalmente se dan situaciones fraudulentas, como la construcción y la hostelería enfocada al turismo, tienen menor peso que en otras regiones. El estudio revela que la evolución del empleo sumergido en Navarra ha ido tradicionalmente en paralelo al empleo legal, de manera que si este aumenta también lo hace el sumergido, a pesar de lo cual, con la crisis, entre 2008 y 2012 se observaba cierta descompensación al decrecer el empleo legal y aumentar el sumergido. Con todo ello, Mauleón y Sardá (2014) cuantificaban para el año 2012 en más de 528 millones de euros el dinero que dejó de ingresar la Hacienda navarra por este motivo y en 46.000 los empleos equivalentes ocultos.

\section{Metodología}

Como se ha indicado, para cada tipo de búsqueda sobre el mercado de trabajo sumergido o informal en Navarra ha sido distinta la metodología utilizada.

\subsection{Metodología para la información generada en Internet}

Para el rastreo, búsqueda y análisis de la información generada en Internet se ha utilizado la técnica del web mining. Esta metodología usa herramientas de minería de datos con el fin de extraer información del contenido de páginas web. Lo más característico aquí es que los sujetos expresan sus opiniones en la Web sin la obligación de someterse a cuestionarios estandarizados o guiones que restrinjan su experiencia a favor de las cuestiones decididas de antemano por un investigador: este método de investigación permite una aproximación naturalista al objeto de estudio, ya que ni el trabajo de campo ni los resultados se ven contaminados por la influencia del investigador (Del Fresno, 2011: 33).

A través de la araña de búsqueda Webrunner, se han examinado conversaciones en Internet (foros, blogs, redes sociales, noticias, sitios, etc.). Una vez encontradas las diferentes conversaciones que surgían a partir de las palabras clave se realizaban distintos procesos para el análisis de esos contenidos, en concreto:

a. Data mining de las opiniones mediante mapeado semántico y árboles semánticos.

b. Clasificado, etiquetado, valoración y análisis de las referencias. 
c. Crackeado de las referencias en opiniones valorativas y opinión movilizada.

d. Análisis actitudinal y posicionamiento valorativo de las opiniones encontradas.

Como refiere Del Fresno (2011: 32), tras el web mining retrospectivo se dispone de los datos (referencias) que conforman el universo. Una referencia es la unidad básica de publicación de información, como puede ser una noticia en un medio de comunicación online, un comentario en un foro, un post en un blog, etc. Esta referencia extraída de la monitorización debe estar en formato texto y contener al menos una de las palabras clave que delimitan el objeto de investigación desde un punto de vista sintáctico. Las referencias deben ser limpias y existentes en la web social sobre las temáticas que son objeto de estudio; de este modo, el universo viene definido por este criterio, así como por la necesidad de que las opiniones en que se descomponen las referencias y comentarios sobre las temáticas de estudio también deben incluir las palabras clave de forma nítida.

Del Fresno (2011: 33) explica que en esta técnica las referencias se pueden desagregar en opiniones, que son la unidad mínima de análisis informacional textual significativa siempre que contengan elementos informativos y/o valorativos relevantes para el objeto de estudio. Para poder abordar la explotación cuantitativa de la información es necesario crear una base de datos para codificar la información. Así, cada referencia y cada opinión es etiquetada o codificada en función de parámetros relevantes para la investigación. Tras esta fase cuantitativa, el modelo propone una fase cualitativa. Se trata de analizar las referencias infotextuales como material empírico tratándolo como una narrativa textual y forma distintiva de discurso para la generación de percepciones útiles. La información extraída es comprendida como una narrativa voluntaria de las expresiones de pensamiento de los sujetos que crean los contenidos infotextuales de forma voluntaria y con objetivos no necesariamente concurrentes.

Para averiguar y definir mejor las tendencias de lo que se busca, la herramienta utilizada ha sido Google Trends, ya que permite visualizar desde 2004 hasta la actualidad qué buscan los internautas en de Google: una marca, una palabra, un producto o una palabra clave como síntesis de una temática. La herramienta aporta información de evolución de indicadores de búsqueda, hasta un máximo de cinco palabras clave. Sus diversas opciones se ilustran en la Figura 1.

Google Trends presenta una serie de gráficas de evolución. En esas gráficas usa un indicador que tiene un valor máximo de cien, pero nunca da el auténtico valor del número de búsquedas para cada clave. El cien de ese indicador es el valor del pico máximo de búsquedas que Google Trends tiene registrado para una clave. A su vez, sobre ese valor calcula el valor del indicador del resto de periodos asociados que coinciden con la búsqueda. Así, la salida de información que proporciona Google Trends, siempre de forma gráfica, es la que muestra la Figura 2.

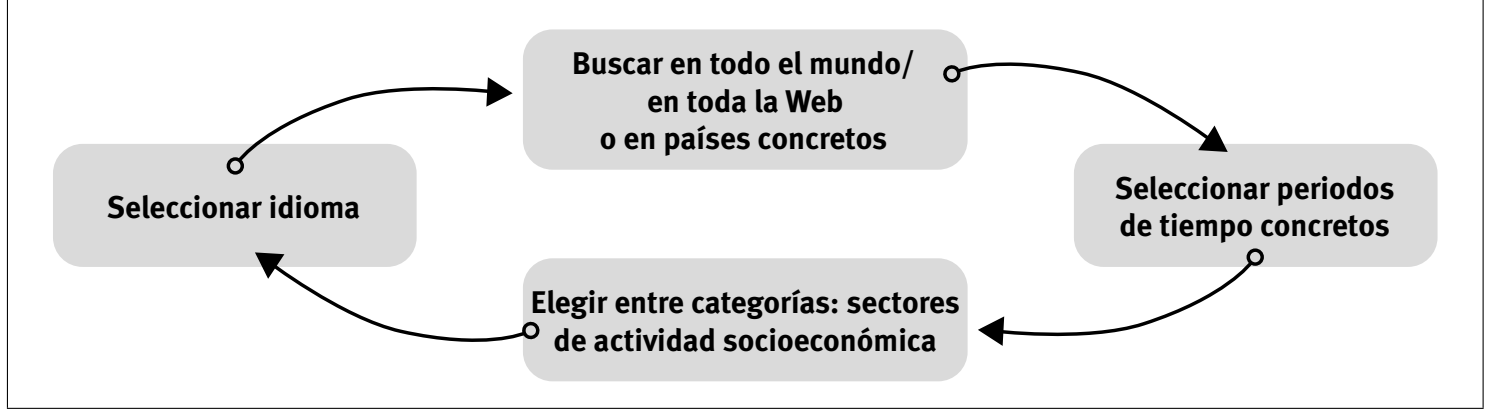

Fuente: Elaboración propia.

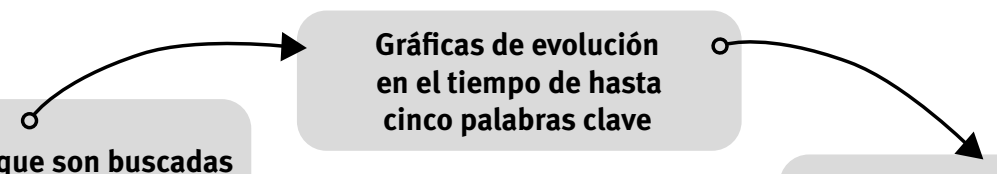

Otras claves que son buscadas a la vez, o por las mismas direcciones que la analizada
Distribución por países, regiones y hasta localidades dentro de un país

Fuente: Elaboración propia. 
En este caso, las palabras claves de rastreo para la búsqueda de conversaciones en medios sociales en Internet fueron: trabajo sumergido, trabajo no declarado, economía sumergida, trabajar sin contrato, trabajar sin factura, trabajo irregular, dinero negro, dinero $B$, ingresos no declarados, fraude fiscal, autónomo, inspección de trabajo y sobresueldo. Cabe señalar que hubo otras tres palabras clave, pero resultaron ser términos con muy poca densidad de utilización: mercado negro, hacienda, declaración de la renta.

\subsection{Metodología para la información escrita en medios de comunicación}

En segundo lugar, para acceder a las noticias en los medios de comunicación escrita se hizo una consulta de la hemeroteca navarra a MyNews, una empresa especializada en el seguimiento de medios, la gestión del conocimiento y el análisis de datos. Esta empresa proveedora de documentación periodística realizó la consulta en base a algunas palabras clave o descriptores que tienen relevancia para la búsqueda y el resultado se presenta en base al tratamiento cuantitativo de los apartados temáticos que han resultado relevantes.

Dicha consulta a la hemeroteca de la base de datos de MyNews se realizó para hallar las referencias con un mínimo de relevancia de los términos de un $10 \%$. Debe indicarse que el porcentaje de coincidencia o relevancia de un artículo va asociado a los términos usados en la ecuación de búsqueda que aparecen en el artículo, por lo que el porcentaje es mayor cuantos más términos aparecen; si un término aparece en el titular o en el subtítulo aumenta su valor, y no todos los términos tienen la misma relevancia: depende de lo comunes que sean. Cuanto menos común es un término, mayor relevancia tiene. Por otra parte, los medios de comunicación se han seleccionado a partir del listado de la Asociación de Periodistas de Navarra y su presencia en la base de datos de MyNews.

La consulta a la hemeroteca en Navarra de los años 2016, 2017 y 2018 se resume en los siguientes cuatro apartados temáticos, y las consiguientes palabras clave o descriptores empleados en la búsqueda:

- Economía sumergida: economía sumergida, trabajo en $B$, trabajo en negro.

- Economía informal: mercado de trabajo, trabajo doméstico, trabajadoras del hogar, empleadas del hogar junto con irregularidad, no regularizado, economía informal, pago efectivo, contratación irregular.

- Inspecciones de trabajo y hacienda: inspecciones ("NEAR trabajo"), inspecciones ("NEAR hacienda, economia sumergida AND trabajo").

- Economía ilegal, informes sobre tráfico de drogas y prostitución: economía ilegal, tráfico de drogas, prostitución, junto con informe.
Tal y como se ha avanzado, el objetivo del análisis es conocer el volumen de noticias y mostrar sus características relacionadas con las palabras clave seleccionadas. Ahora bien, en base a la metodología expuesta por Igartua, Muñiz y Cheng sobre las aportaciones empíricas y metodológicas de la teoría del encuadre noticioso "se examinan las noticias desde un punto de vista abierto y se intenta revelar el conjunto de posibles encuadres, sin tener prefijados de antemano un número determinado" (Igartua et $a l$., 2005: 150). Por tanto, y aunque la búsqueda se centre en los ámbitos y palabras clave, se desconocen a priori las particularidades que puedan emerger para el análisis de las noticias.

\section{Resultados y discusión}

\subsection{Las alusiones al mercado de trabajo sumergido en los medios sociales}

El resultado de la búsqueda de referencias en Internet se tradujo en un total de 903 conversaciones $\mathrm{u}$ opiniones mantenidas en los medios sociales acerca del mercado de trabajo sumergido o "en B" entre 2016 y 2018, siempre ligadas a que en algún parámetro de la conversación se aludiera a Navarra o que la IP desde donde se emitía la opinión fuera de Navarra. Respecto al rastreo de las palabras clave relacionadas con el mercado de trabajo sumergido o “en B" y su campo semántico de actividad, durante el periodo seleccionado para el estudio podemos observar que "fraude fiscal" es la palabra clave más utilizada de todas con diferencia y es la que reporta más resultados en la evolución de búsquedas. Dicho de otro modo, "fraude fiscal" es la referencia en Internet sobre esta temática. En el resto de palabras clave su uso en búsquedas desciende, si bien es “dinero B" la que obtiene una presencia algo más notoria que el resto entre el conjunto de rastreadas.

En cuanto a la tipología de los medios sociales en los que se conversa sobre esta temática y la intensidad con que se habla, la opinión movilizada trasluce que los medios de comunicación (online y offline) y los agregadores de opinión destacan frente a los medios solo online y blogs a la hora de encontrar opiniones sobre el mercado de trabajo sumergido y las diferentes palabras clave rastreadas.

Entre los medios con más profusión de conversaciones, por orden de mayor a menor, encontramos a la agencia Europa Press (europapress. es, con el 7,3\%), eldiario.es ( $5 \%$ ), elpais.com (4,8\%), lainformacion.com (3,2\%), noticiasdenavarra.com $(3,2 \%)$, elmundo.es $(2,7 \%)$, cadenaser.com $(2,3 \%)$, diariodenavarra.es (2,3\%) y elespanol.com (2,3\%); el resto están por debajo del $2 \%$.

Por su parte, si se analiza el sentimiento de las 903 conversaciones identificadas y etiquetadas, se establece el posicionamiento valorativo general del conjunto de las opiniones. Así, prevalece claramente un sentimiento negativo hacia el mercado de trabajo 
Figura 3. Tipología de los medios sociales con opiniones sobre el mercado de trabajo sumergido

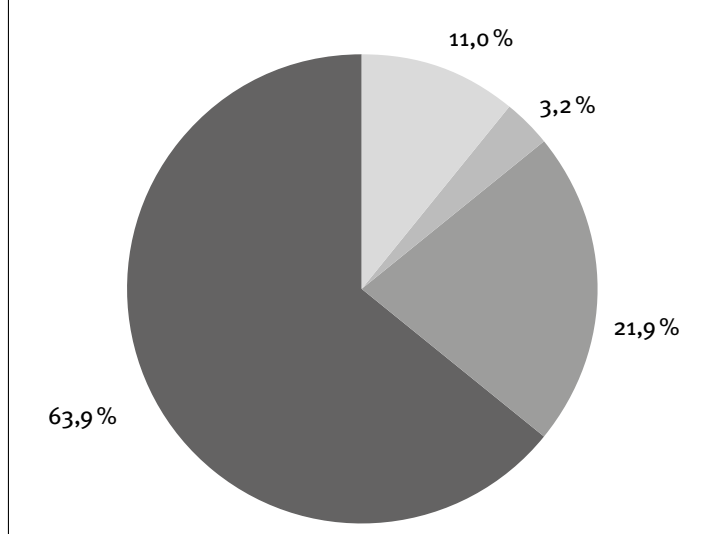

Medio de comunicación solo online Blog

Agregadores de opinión

Medios de comunicación online y offline

Fuente: Elaboración propia.

sumergido o en B (el $65 \%$ del total), algo más de una cuarta parte de las valoraciones muestran valoraciones positivas $(26,4 \%)$, mientras que el $8,6 \%$ son de carácter neutro.

En este sentido, el análisis del posicionamiento valorativo de las opiniones por tipología de medio social indica que son los medios de comunicación solo online los que mejor valoración muestran hacia el mercado de trabajo sumergido (30,7\%). Por el contrario, los comentarios que se hacen en los blogs (aunque su presencia sea algo menor) respecto al mercado de trabajo sumergido tienen un marcado carácter negativo (83,3\%). La precariedad en los contratos, el fraude y los abusos detectados en el mercado laboral, así como las recientes movilizaciones sociales realizadas con relación a las pensiones y los derechos de la mujer pueden haber contribuido a ello.

Otro examen interesante se refiere a los términos que se mencionan junto con "mercado de trabajo sumergido". Del conjunto de términos citados en las conversaciones se extrae que "empleo sumergido" es el que aparece con mayor presencia en la Red y en los medios sociales durante el rastreo realizado; esta es la marca fundamental porque supone el $63,7 \%$ de las menciones en todas las conversaciones en que aparecen las palabras clave rastreadas.

La mención a "precariedad laboral” utilizada por los internautas a la hora de hablar del mercado de trabajo sumergido es de un $9,5 \%$ en las conversaciones. Asimismo, aparecen menciones al Plan de lucha contra el fraude laboral y la Seguridad Social, así como sobre la Comisión de lucha contra el fraude fiscal, del orden de un $5,2 \%$ y un $5,1 \%$, respectivamente, cuyo posicionamiento en más de un $60 \%$ de los casos es de carácter positivo. Por último, también hay algunas referencias genéricas a las actuaciones realizadas por el Gobierno de España ( $5,1 \%$ de las menciones), el Gobierno Vasco (1,8\% de las menciones), la Unión Europea (1\%) y el Gobierno de Navarra (0,8\%).

A continuación, se presentan las representaciones gráficas en forma de gráfico de burbujas y nubes temáticas de estas masas de información que se mencionan junto con "mercado de trabajo sumergido".

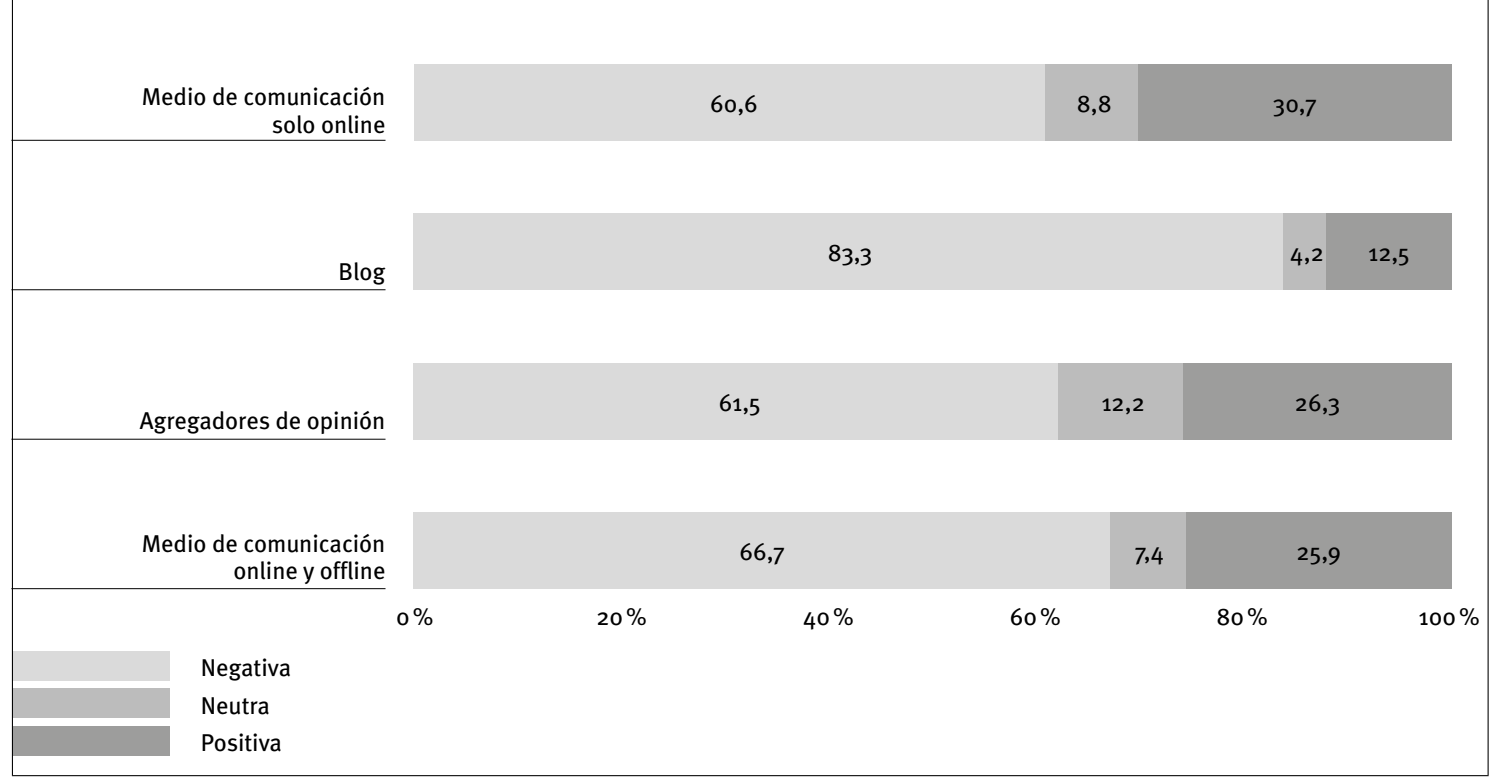

Fuente: Elaboración propia. 


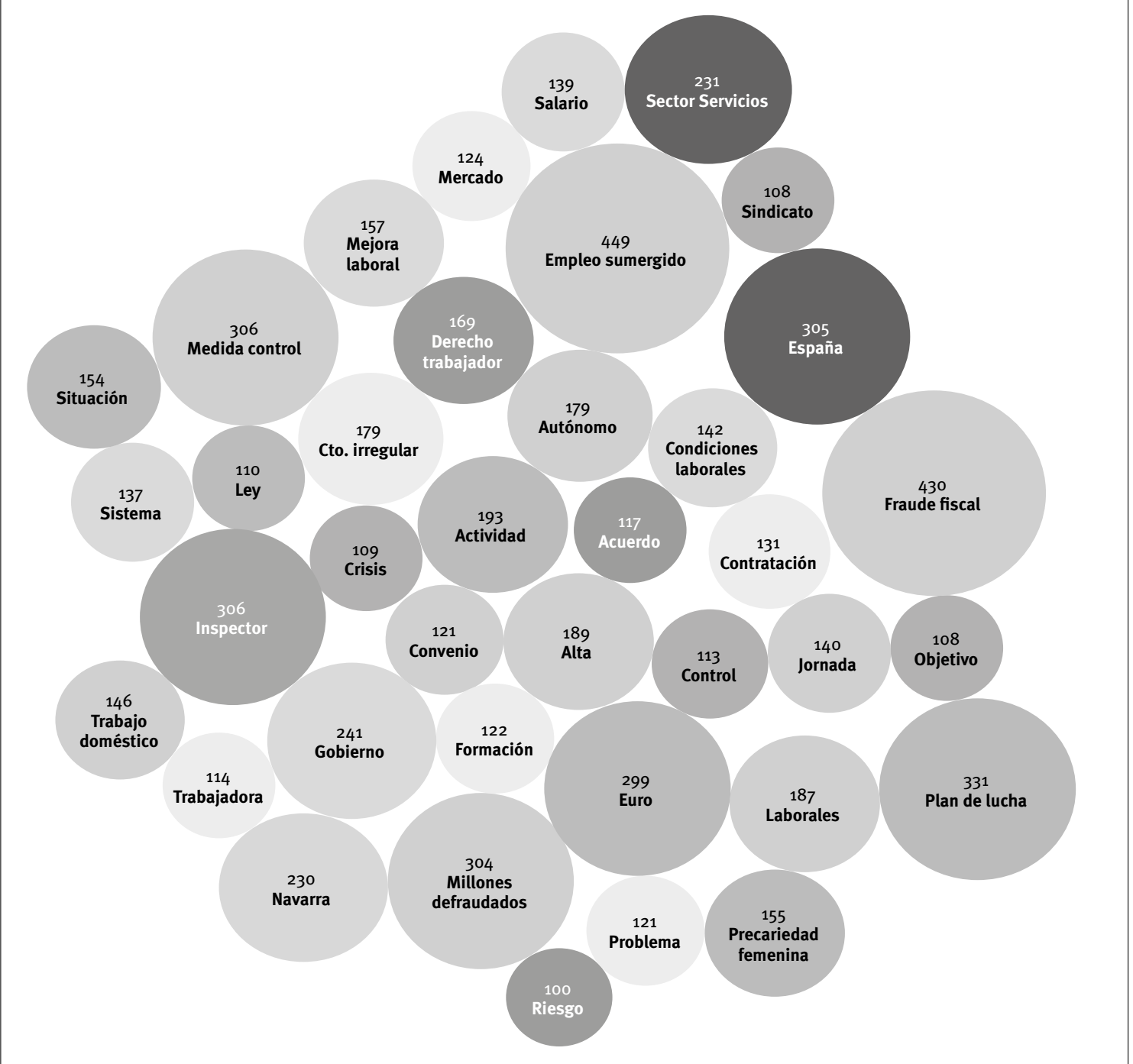

Fuente: Elaboración propia.

Otra forma de ver y analizar los contenidos asociados en las conversaciones es mediante las nubes de etiquetas o nubes semánticas, que muestran, en función del tamaño de las palabras representadas, la mayor o menor intensidad o frecuencia de aparición o presencia en las conversaciones.

Obviamente, “empleo sumergido" es la palabra clave dominante en intensidad de presencia en los contenidos. En el análisis de las 25 palabras o temáticas más frecuentes y recurrentes, se observa que algunos conceptos abundan más en las conversaciones. Son los términos que contribuyen con mayor intensidad a construir o formar los el mercado de trabajo sumergido. Estos términos clave con los que se identifica la economía sumergida son: los autónomos, el fraude, la precarización femenina, los inspectores, las actuaciones del Gobierno, el plan de lucha contra el fraude fiscal, la irregularidad de los contratos, los millones de euros defraudados a la Seguridad Social, etc.
Al respecto, cabe señalar que las temáticas de las que más se habla en la web social en relación con nuestro objeto de estudio tienen relación sobre todo con el fraude fiscal (39,8\%), los empleos irregulares $(13,4 \%)$, la precariedad en el trabajo desarrollado por mujeres (12,3\%), la importante presencia de empleo sumergido en el sector servicios, el ámbito de los autónomos y la agricultura (9,4\%), el crecimiento del empleo sumergido durante los últimos años (3,4\%), la importancia de generar empleo de calidad (3,3\%), la dificultad de luchar contra el fraude al considerarlo casi un atributo propio de la economía española $(2,7 \%)$, y la problemática generada en el ámbito de las cotizaciones y pensiones (2,7\%), mientras que el resto de temas no superan el $2 \%$.

En lo concerniente a la valoración del "mercado de trabajo sumergido" por categorías temáticas y teniendo en cuenta que la valoración general sobre este tema es de tendencia negativa, no es de extrañar que dicha valoración sea similar y predomine la crítica. En total, son cinco las grandes 
Figura 6. Mapa semántico del total de opiniones (nube de etiquetas, 25 palabras)

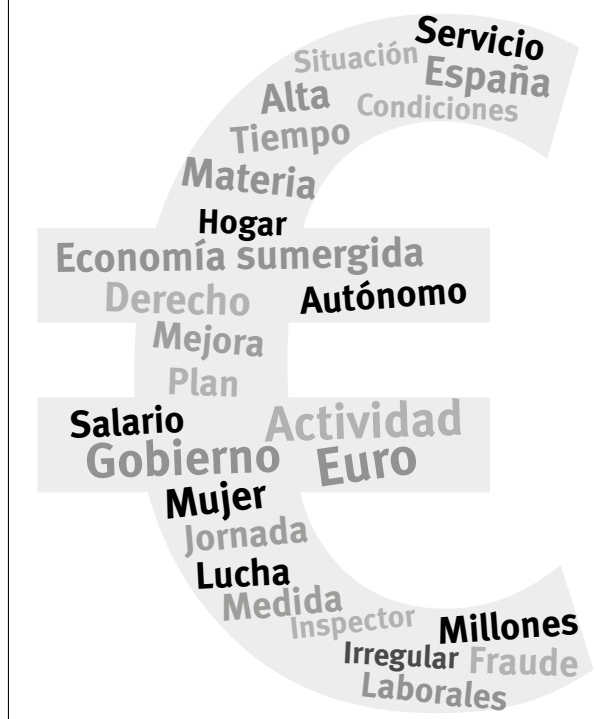

Fuente: Elaboración propia.

categorías que aparecen en las conversaciones: fraude fiscal, precariedad laboral, sectores, inspección y protección del trabajador. Las categorías con más opiniones positivas son aquellas que tienen que ver con las inspecciones realizadas y la lucha emprendida desde las instituciones contra el fraude fiscal. En cambio, las categorías con más opiniones negativas son aquellas que tienen que ver con los sectores donde más presente está el fraude fiscal, la precariedad laboral existente en el mercado y el fraude fiscal implantado en la sociedad actual.

Por orden de mayor citación de categorías, la información relativa al fraude fiscal centra el discurso de un $50,6 \%$ de las opiniones etiquetadas. Aun con una mayoría de opinión crítica y de censura ante este tipo de prácticas que resultan altamente cuestionadas, es de destacar también que un tercio de las opiniones son positivas cuando se habla de la lucha contra estas conductas. La información relativa la precariedad laboral constituye la segunda categoría de temáticas en el discurso, con un $31,1 \%$ de las opiniones etiquetadas. La posición valorativa manifestada es negativa mayoritariamente, ya que responde a informaciones que tienen que ver con la inestable, pobre y frágil situación que presenta el mercado laboral y las relaciones contractuales en él desarrolladas. La necesidad de acabar con esta precariedad laboral y generar empleos de calidad es el único nodo aquí que avala las valoraciones de corte positivo.

En tercer lugar, por categorías temáticas, la información relativa a los sectores en los que más se detecta o está presente la economía informal cuenta con un 13,9\% de las opiniones etiquetadas. De nuevo la mayor parte son muy negativas, sobre todo por tratarse de casos de fraude, desfalco y prácticas engañosas que resultan altamente cuestionadas. El cuarto aspecto que centra las conversaciones mantenidas en la red, aunque con menor presencia que los casos anteriores $3,4 \%$ de las opiniones etiquetadas) es la información relativa a la gestión de las inspecciones realizadas en este ámbito. El sentimiento valorativo manifestado por los opinantes en las conversaciones analizadas es positivo, sobre todo por las acciones emprendidas contra el fraude (como la posibilidad de realizar denuncias anónimas por la ciudadanía) y los resultados positivos obtenidos, bien en cuanto a recuperación de fondos defraudados, regularización de contratos de trabajo, detención de personas inmersas en actividades fraudulentas, etc.

Finalmente, la categoría con un menor porcentaje de informaciones versa sobre la protección del trabajador, un $0,9 \%$ de las opiniones etiquetadas. Aquí la mayor parte de las opiniones son neutras, ya que son más conversaciones informativas en los medios de comunicación que valorativas en sí mismas.

Figura 7. Posicionamiento valorativo sobre "mercado de trabajo sumergido" por categorías temáticas (\%)

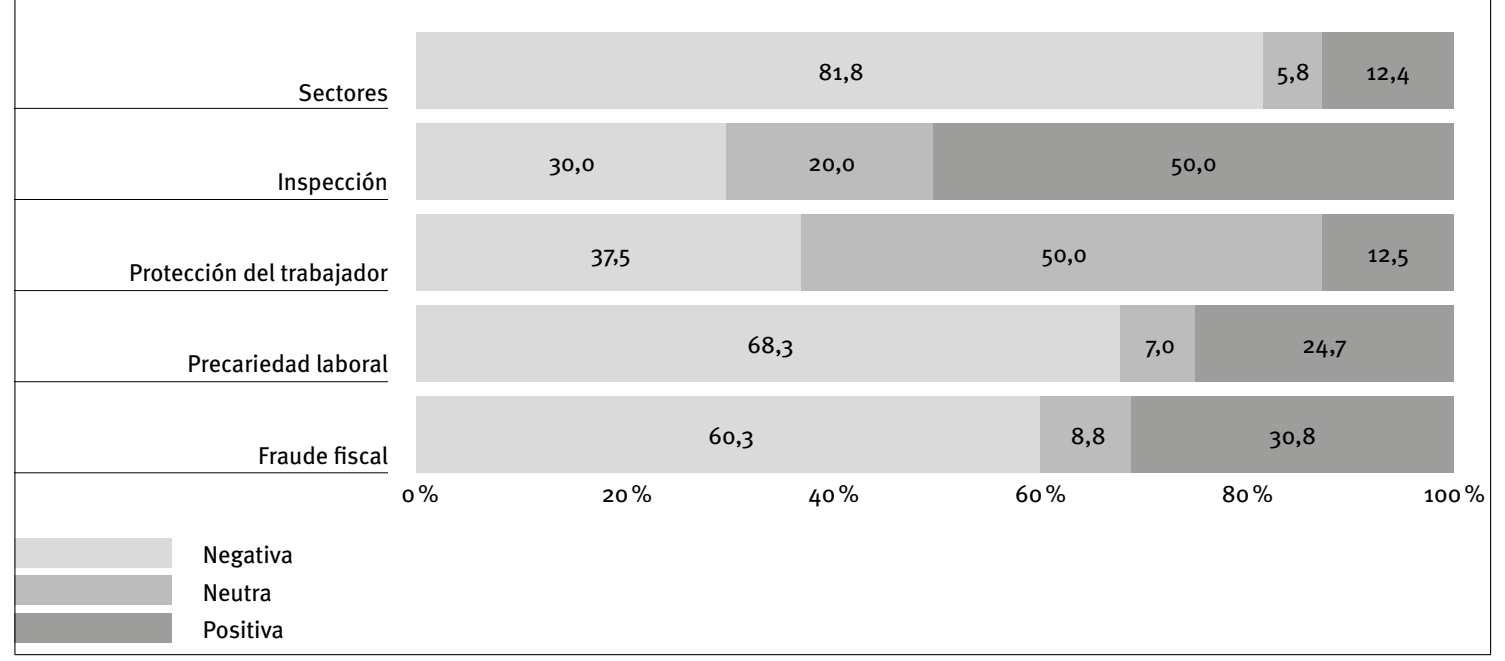

Fuente: Elaboración propia. 


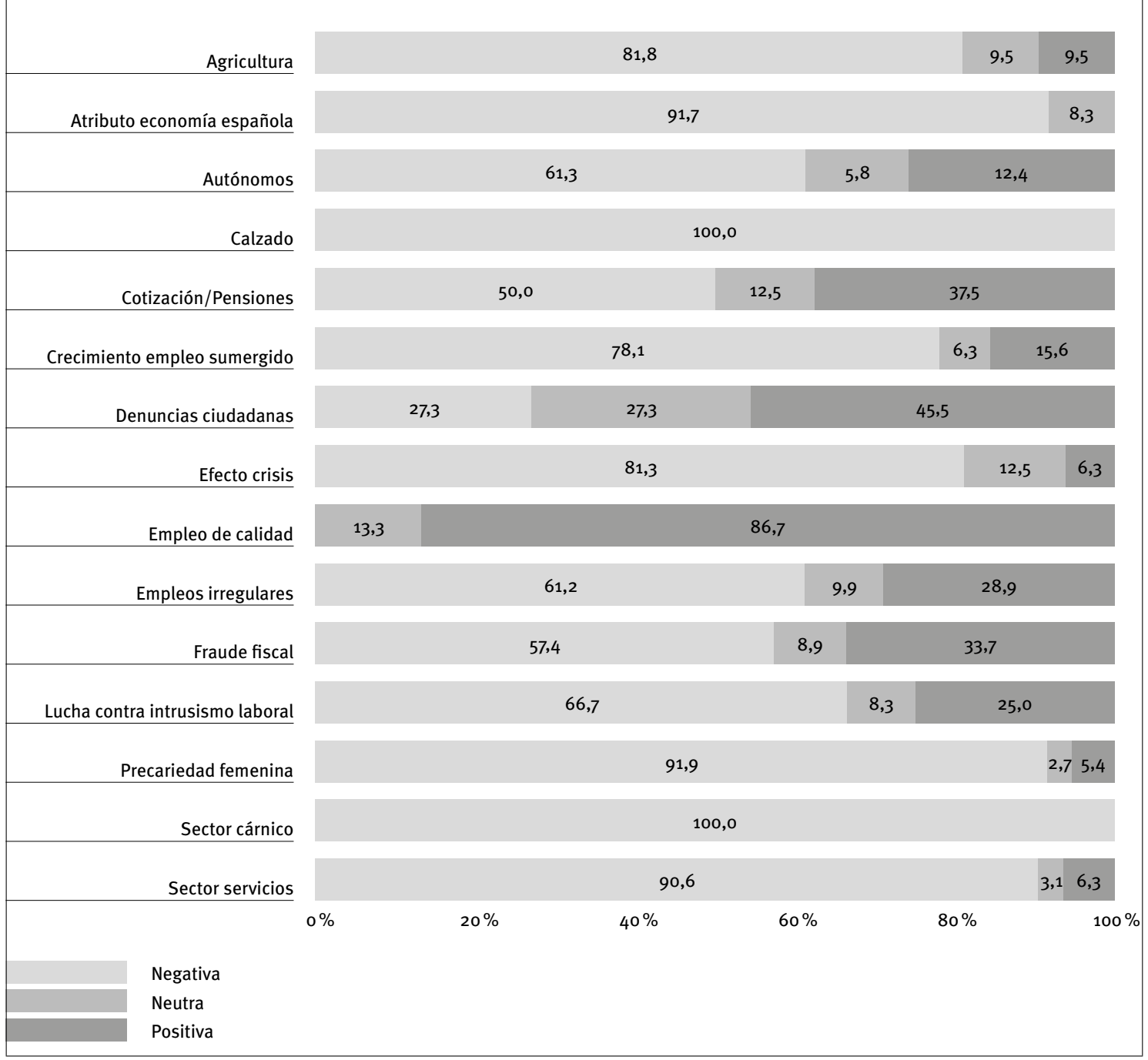

Fuente: Elaboración propia.

Por último, se recoge el posicionamiento valorativo de las principales subcategorías temáticas en función de la visibilidad e intensidad con la que han aparecido. Aquí se observa que el empleo sumergido en el sector del calzado, cárnico y de servicios (con mucha menor implantación en Navarra en cualquier caso para los tres sectores), la precariedad femenina y la consideración de que defraudar es un atributo propio de la economía española poseen una opinión valorativa con mayor tendencia negativa.

\subsection{Las alusiones al mercado de trabajo sumergido en la prensa escrita}

El resultado de la consulta a la hemeroteca navarra para buscar noticias en prensa escrita obtuvo 376 referencias como base para esta aproximación a su tratamiento. Entre las 376 referencias del periodo de la búsqueda, destaca el elevado número de referencias de Diario de Navarra (160) y de Diario de Noticias (141). El número de referencias recogidas en el diario Gara (65) se reduce notablemente en comparación con los dos diarios anteriores, pero aún es menor el de otros medios de comunicación de tirada nacional (10 referencias) incorporadas a la búsqueda sobre la economía sumergida en Navarra (El País y El Mundo, y los diarios sobre economía Cinco Días y Expansión).

Durante el proceso de revisión de las referencias de la muestra, se descartó el $53 \%$ (208 referencias). La mayoría fueron eliminadas al ser referencias duplicadas en las ediciones comarcales de algún periódico y otras porque se trataba de noticias relacionadas, generalmente, con escándalos de fraude y corrupción política y empresarial, aunque también se descartó un elevado número de referencias que no guardaban interés por hallarse en secciones de la prensa sin interés para la investigación.

Por lo tanto, el listado de referencias, tras el proceso de descarte detallado, alcanza un número total de 185 referencias de interés para analizar el fenómeno de la economía sumergida y las actividades buscadas 
en esta aproximación. Cabe destacar el desigual número de noticias por medios: 90 referencias de Diario de Noticias; 69 de Diario de Navarra; 16 de Gara; y las diez referencias remitidas sobre medios nacionales de los años 2016, 2017 y 2018.

Tabla 1. Muestra de prensa seleccionada a partir de las referencias remitidas

\begin{tabular}{|l|c|c|c|c|c|}
\cline { 2 - 6 } \multicolumn{1}{c|}{} & $\begin{array}{c}\text { 'Diario de } \\
\text { Noticias' }\end{array}$ & $\begin{array}{c}\text { 'Diario de } \\
\text { Navarra' }\end{array}$ & 'Gara' & $\begin{array}{c}\text { Medios } \\
\text { nacionales }\end{array}$ & Total \\
\hline 2016 & 45 & 32 & 4 & 1 & 82 \\
\hline 2017 & 34 & 27 & 7 & 1 & 69 \\
\hline 2018 & 11 & 10 & 5 & 8 & 34 \\
\hline Total & 90 & 69 & 16 & 10 & 185 \\
\hline
\end{tabular}

Fuente: Elaboración propia.

A continuación se detallan los tres tipos de análisis realizados en base a estas 185 informaciones: análisis temático de las referencias periodísticas, por titulares y contenidos, y por el origen de las referencias. En primer lugar, en cuanto al análisis de las temáticas principales detectadas en las referencias periodísticas, estas se encuadran en tres segmentos principales: el ámbito fiscal $(84$ referencias), el ámbito laboral (83 referencias) y el ámbito de la economía ilegal (18 referencias).

Antes de diferenciar las referencias periodísticas de la muestra en diversas temáticas, cabe introducir una relevante nota aclaratoria sobre el carácter polisémico del concepto de "economía sumergida" que dificulta su etiquetaje. Las diferentes acepciones y concepciones del término son notables y condicionan una distribución nítida de las temáticas en las que analizar las referencias.

Dentro del ámbito fiscal (84) destaca el elevado número que analizan el fraude fiscal (68 referencias). En el relativamente reducido número de referencias restantes no relacionadas con el fraude fiscal (14), se analizan aspectos de la tributación, como las solicitudes de bajadas de impuestos por parte de los empresarios o temas relacionados con el ahorro, los presupuestos y la deuda pública. En el caso de las noticias de este primer grupo de referencias periodísticas sobre el ámbito fiscal, se trata de informaciones extensas y elaboradas que dan cuenta de algún estudio, informe o inspección (67\% de las referencias), generalmente publicado por la Administración. El tono de las noticias es neutro, claramente informativo, y puede percibirse cierto nivel de especialización mayor que en el caso de las referencias relativas a los restantes ámbitos. Por último, debe destacarse que los artículos de opinión sobre el ámbito fiscal vienen mayoritariamente firmados por expertos y docentes; y que siete editoriales de distintos medios analizan problemáticas relacionadas con este ámbito.

Si pasamos al segmento del ámbito laboral (83 referencias), se han hallado 16 sobre problemáticas de las empleadas del hogar y las actividades laborales remuneradas de ese entorno, así como tres referencias sobre aspectos relacionados con el sector de los cuidados (en total, ocho noticias ofrecen datos relativos al elevado empleo irregular de este sector en Navarra). Las referencias sobre el empleo irregular agrupan la mayoría de los textos restantes (18 referencias), seguidas por las referencias que tratan asuntos relacionados con las relaciones laborales (11). Este grupo de referencias relativas a aspectos del ámbito laboral se complementa con otras relacionadas a las pensiones (8 referencias), la seguridad y la salud laboral (7), el régimen especial de autónomos (7) y otras que afectan o combinan varios temas.

Las personas inmigrantes tienen una presencia transversal y relevante en este ámbito laboral. Por una parte, se menciona al numeroso colectivo de mujeres que trabajan como empleadas del hogar en este sector. Por otra parte, se han hallado cuatro noticias que relacionan a las personas inmigrantes con la Renta Garantizada; en dos referencias critican el supuesto efecto llamada de esta prestación social, y otras dos defienden al colectivo y el valor de la prestación.

Cabe destacar que ocho de las referencias de este apartado de noticias toman como base informativa las inspecciones de trabajo, y otras 28 referencias se basan en informes y estudios. Este dato muestra que casi la mitad de las referencias ( $43 \%$ ) se basan en inspecciones de trabajo, informes y estudios, y que el tratamiento de las noticias sigue teniendo un carácter informativo, aunque las referencias que toman como base las fuentes sindicales dan voz a las reivindicaciones de diversos colectivos, por ejemplo, al de las empleadas del hogar. La participación del colectivo de mujeres inmigrantes en el sector informal tiene un tratamiento correcto (aunque resulta dudoso en algún artículo de opinión), o al menos no pueden destacarse informaciones de carácter negativo o discriminatorio.

Por último, en cuanto al ámbito de la economía ilegal, la búsqueda de noticias sobre prostitución (11 referencias) y drogas (7 referencias) estaba limitada a recopilar únicamente referencias periodísticas basadas en informes o estudios. A pesar de tratarse de una búsqueda de estas características, el reducido número de referencias recopiladas tratan sobre cuestiones en las que es difícil discernir el alcance de la economía informal en esta área, y son emitidas con un carácter más bien neutro.

En segundo lugar, en cuanto al análisis de titulares y contenidos, en el siguiente gráfico se muestran los titulares y contenidos de las temáticas con mayor prominencia de los tres ámbitos anteriores. El objetivo es conocer cuáles son los aspectos más frecuentemente tratados por la prensa. Por lo que respecta al primer ámbito, se presenta la frecuencia de referencias halladas en relación a la lucha y control sobre el fraude fiscal de los dos Gobiernos, 
pero también se analiza la frecuencia de titulares y contenidos sobre el fraude del alquiler. Dentro de las referencias del ámbito laboral, se presentan los porcentajes temáticos de las elevadas referencias sobre las trabajadoras del hogar; la calidad y la precariedad del empleo; y el porcentaje relativo a la presencia de inmigrantes en titulares y contenidos, debido a su elevada participación en el empleo irregular y el trabajo doméstico. En el tercer ámbito, el de la economía ilegal, se presentan los porcentajes de referencias recopiladas acerca de las temáticas sobre prostitución y drogas, que den cuenta de algún informe.

Las noticias referentes a la lucha contra el fraude fiscal de los Gobiernos foral y estatal copan un $12 \%$ de los titulares de toda la muestra de esta aproximación, y los contenidos alcanzan un $22 \%$ de las referencias periodísticas analizadas en este examen que abarca tres años. Dentro del ámbito fiscal, también destaca la frecuencia de titulares y noticias sobre el fraude en los alquileres, sobre todo los de uso turístico en Pamplona. En el ámbito laboral es lógico que por el condicionamiento de la búsqueda adicional sobre economía informal se haya encontrado un elevado número de referencias. Es relativamente reducido el número de referencias sobre precariedad y calidad del empleo dentro este ámbito laboral de la economía sumergida. Destacan, de una manera lógica, las referencias periodísticas sobre el colectivo de mujeres inmigrantes. En el caso de las referencias sobre drogas y prostitución, estos resultados están condicionados por su necesidad de estar relacionados con algún estudio o informe.

Por último, respecto al análisis por el origen de las referencias, el $29 \%$ se encuentra en las noticias de la sección de "Economía y Trabajo", el $24 \%$ son artículos de opinión y editoriales, un $19 \%$ se halla en la sección de "Sociedad", un $10 \%$ en las secciones "Vecinos" o "Local”, un $10 \%$ en "Miscelánea”, un $6 \%$ en "Nacional" y un $2 \%$ en "Cultura". La mayoría de noticias que tratan temáticas de Navarra agrupa 105 referencias, las noticias de ámbito nacional servidas por agencias son 37, y las referencias agrupadas en el segmento de editoriales y artículos de opinión alcanzan las 43. Entre los artículos de opinión resulta destacable la colaboración de expertos y docentes, además de los textos escritos por representantes políticos, de sindicatos, asociaciones, fundaciones y los propios periodistas.

\section{Conclusiones}

Este artículo recoge una aproximación al tratamiento mediático y online acerca del mercado de trabajo sumergido o informal en Navarra. La novedad de este estudio se debe a la doble metodología empleada. En primer lugar, a través del rastreo, búsqueda y análisis de la información generada en los medios sociales en Internet y, en segundo lugar, mediante la consulta a la hemeroteca de los medios de comunicación escritos y el correspondiente análisis de las noticias. El alcance temporal comprende de manera retrospectiva un total de 36 meses tanto para el análisis en redes sociales como para la búsqueda de noticias en medios de prensa escrita (desde enero de 2016 hasta diciembre de 2018).

En primer lugar, respecto a las búsquedas realizadas en Internet, "Fraude fiscal” es la palabra clave más utilizada y la que reporta más resultados en la evolución de búsquedas, frente a otras como "dinero b", "economía sumergida", "trabajo precario", "trabajo irregular”, “empleo sumergido”, etc. Los

Figura 9. Titulares y contenidos de mayor frecuencia en la muestra de prensa escrita (\%)
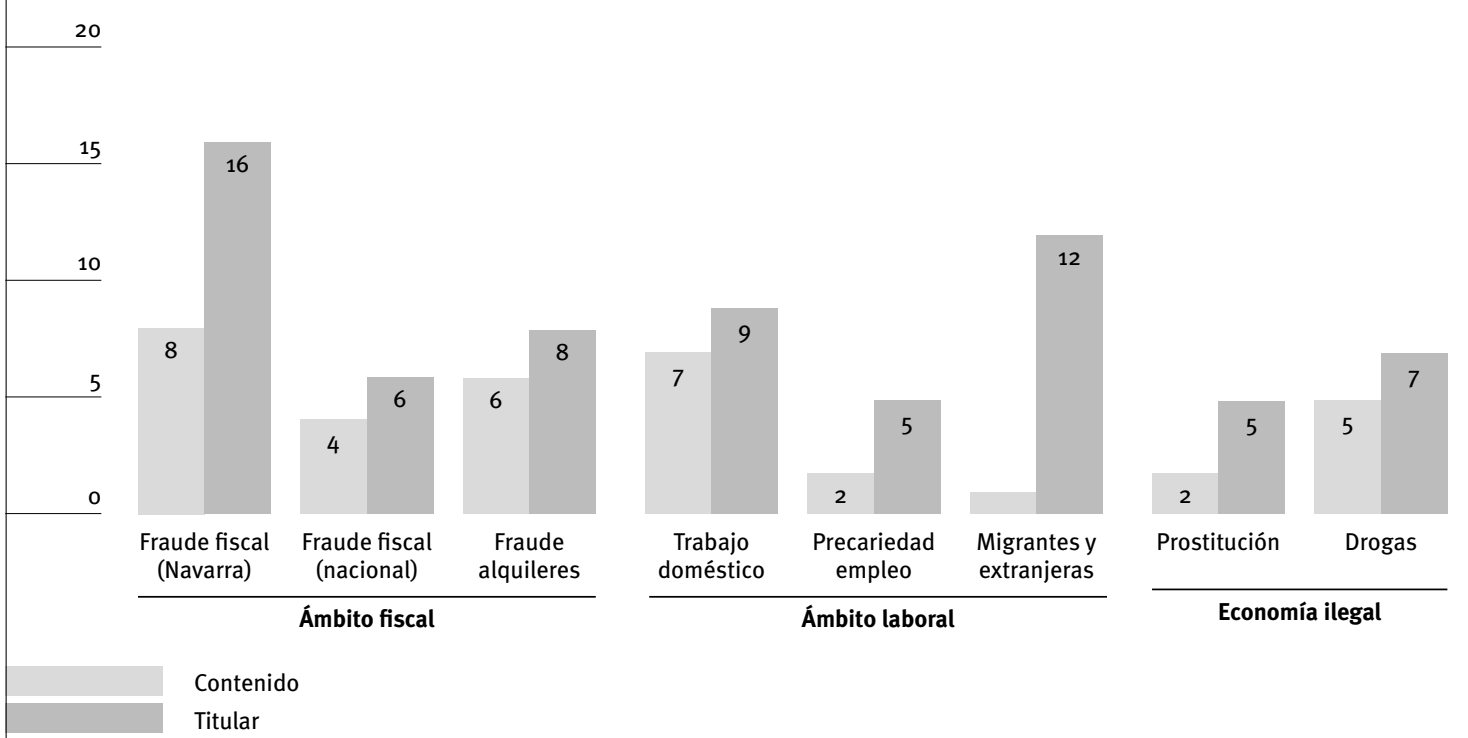

Fuente: Elaboración propia. 
medios de comunicación online y offline son los principales medios donde se opina sobre el mercado de trabajo sumergido, generalmente porque dan difusión a las noticias que sobre esta palabra clave se reproducen en las redes. La presencia en redes sociales es inexistente y no se presenta como un canal de conversación con los usuarios internautas opinantes. Así, la estrategia en medios sociales ha sido más bien informativa, dándose difusión a las noticias producidas sobre esta temática y a las reacciones y consecuencias generadas por las acciones desarrolladas en torno a ella. A pesar de la creciente relevancia de las redes sociales, no se ha percibido que se produzca un intercambio de conversaciones con los usuarios opinantes en los diferentes medios, ni que se realice una gestión de esa información con los internautas opinantes.

La temática de la que más se habla en el mundo online en relación a este ámbito es el propio empleo sumergido, en cuanto a la gran presencia que tiene hoy en día en el mercado laboral y la sociedad, sobre todo en determinados sectores, la precariedad laboral asociada a él y el importante fraude fiscal que se produce como consecuencia de su desarrollo y expansión. El contenido de las informaciones emitidas, pese a tener un perfil más bien periodístico, provocan que la mayor parte de las opiniones que se generan en relación a ellas posean una valoración negativa y crítico, lo que a su vez provoca que el impacto que se crea con estas conversaciones y las posibles retroalimentaciones que se den, expandan este sentimiento de rechazo o reprobación.

El conjunto de temáticas que tienen que ver de un modo más directo con el mercado de trabajo sumergido abarcan un gran porcentaje de valoraciones negativas, relacionadas sobre todo con el fraude fiscal, los empleos irregulares, la precarización laboral femenina, la gran presencia de economía sumergida en el sector servicios y el de los autónomos, la dificultad de luchar contra el fraude por considerarlo prácticamente un elemento integrado en el ADN de la economía española, etc. Este tipo de noticias en las redes actualmente suponen más de un $70 \%$ de las opiniones etiquetadas, frente a aquellas que tienen que ver con las inspecciones y los controles realizados, que no llegan a alcanzar un $25 \%$. Se puede pensar que promover una imagen más positiva dentro de este ámbito y una mayor generación de hilos de conversación con sentimiento positivo podría producirse con un incremento en la difusión de información y noticias que potencien las acciones desarrolladas en la lucha contra el fraude y en la mejora de las condiciones del mercado laboral, tanto en los aspectos que competen al Gobierno e instituciones, como en las que competen al entramado empresarial.

Las categorías de opiniones más positivas son aquellas que tienen que ver con las inspecciones y macrooperaciones de control realizadas desde las Administraciones e instituciones, con el fin de erradicar el fraude fiscal y las prácticas irregulares en el mercado laboral. Las categorías con más opiniones negativas, sin embargo, son aquellas que ponen en evidencia no solo los sectores en los que se encuentra más presente el empleo sumergido, sino además aquellos en los que se encuentra presente de un modo natural y más interiorizado, como si fuese una práctica normalizada, la precariedad laboral existente y el fraude fiscal implantado en la sociedad actual.

En segundo lugar, respecto al análisis de las referencias aparecidas en los medios de comunicación escritos, como en el caso anterior las noticias relacionadas con la lucha contra el fraude fiscal generado por la economía sumergida destacan de entre todas las referencias analizadas. Además de que una de cada cinco referencias tenga relación con la inspección y el control de Hacienda, puede apreciarse cierto nivel de especialización en ellas. En el caso de este tipo de noticias del ámbito de la Comunidad Foral de Navarra, las noticias vienen generalmente firmadas por sus autores, pero no es el caso de las noticias de ámbito nacional, debido a que estas vienen servidas por agencias de noticias. La cuestión de que las noticias publicadas tengan la firma de una agencia no tiene que implicar una merma en su calidad, pero indica que algunas temáticas concretas sean siempre redactadas por periodistas con cierto nivel de especialización, mientras que otro tipo de información se cubre a partir de ese recurso. En su tesis doctoral, Alberto Durán analiza la especialización de los periodistas en el ámbito de la inmigración, donde observa que es un recurso muy habitual de la prensa escrita, como también es habitual recurrir a ONG, por ejemplo, para ofrecer información especializada. Durán (2011: 263) afirma que "mientras que los temas económicos deben ser abordados por especialistas, se acepta que otros relativos a aspectos menos valorados socialmente puedan ser tratados por periodistas 'todoterreno', como es el caso de los temas sociales que no requerirían, por ejemplo, de sociólogos".

En los antecedentes teóricos se anticipaba el carácter no polémico de los conceptos analizados en relación con la función de establecimiento de la agenda por parte de estos medios. El análisis confirma esa hipótesis y puede afirmarse que no se han hallado encuadres polémicos en el conjunto de noticias analizadas en esta aproximación y las noticias de la muestra tienen un tono informativo y neutro. En el caso del establecimiento de la agenda, no parece que ni la prensa navarra ni la nacional incidan en esa función, y en todo caso puede afirmarse que la elaboración de las noticias viene condicionada por la publicación de datos, informes o estudios de las Administraciones Públicas y de otro tipo de entidades. La muestra recoge ejemplos de noticias publicadas por los medios analizados en las mismas fechas y con titulares muy similares.

Con todo ello, este tipo de análisis entendemos que arroja luz sobre cómo concienciar socialmente 
sobre este problema, que desde luego no es menor en nuestra sociedad. Hasta ahora el enfoque para disminuir el efecto de la economía sumergida ha ido en el sentido de propuestas judiciales y tributarias, el aumento de las inspecciones laborales o el fomento de sectores plenamente regulados (Mauleón y Sardá 2014), pero también estos mismos autores proponían la puesta en marcha de medidas de concienciación social, como puede ser el tener una estrategia de comunicación clara y positiva en este sentido. De este modo, parece relevante mostrar e informar con mayor presencia en las redes sociales y en los medios escritos de la eficacia en la lucha y control contra el fraude, así como de las estrategias que se están desarrollando desde las instituciones y administración para frenar o combatir la precariedad laboral.

Actualmente, la sociedad manifiesta un discurso cada vez más crítico con las actuaciones irregulares y con los distintos tipos de fraude realizados por representantes políticos, financieros, empresariales, etc., máxime cuando durante los últimos años se han incrementado las situaciones de precariedad laboral. La crisis ha generado un aumento de muchos problemas entre los trabajadores. Frente a todas estas informaciones negativas que llevan a que las opiniones sobre el empleo sumergido oscilen hacia la crítica y el descrédito, parece necesario potenciar aquellas informaciones que pongan en valor la implementación de una legislación más estricta, la vigilancia en el fraude, la inversión en recursos para la vigilancia de las actuaciones de este tipo, la apuesta de los Gobiernos por desarrollar planes de trabajo digno de cara al futuro, etc. Fomentar este tipo de conversaciones e hilos pueden favorecer una mejor imagen de las instituciones y una sensación en los opinantes de que existe una escucha y alineamiento hacia las demandas y necesidades de la sociedad actual. 


\section{Bibliografía referenciada}

CANEL, M.J. (1999): “El País, ABC y El Mundo: tres manchetas, tres enfoques de las noticias", ZER Revista de Estudios de Comunicación, 6, págs. 97-117.

CANEL, M.J.; LLAMAS, J.P. y REY, F. (1996): “El primer nivel del efecto agenda-setting en la información local: los 'problemas más importantes' de la ciudad de Pamplona”, Comunicación y sociedad, Vol IX, № 1 y 2, págs. 17-37.

CONSEJO ECONÓMICO Y SOCIAL DE ESPAÑA (1999): Informe, por propia iniciativa, sobre la economía sumergida en relación a la quinta recomendación del Pacto de Toledo, Sesión ordinaria del Pleno de 21 de abril de 1999 , Consejo Económico y Social de España.

DEL FRESNO, M. (2011): "Cómo investigar la reputación online en los medios sociales de la web 2.0", Cuadernos de Comunicación Evoca, vol. 5, n. 1, págs. 29-33.

DURÁN, A. (2011): El perfil del periodista vasco y su formación el ámbito social de la inmigración. Tesis doctoral, Bilbao, Universidad del País Vasco.

GOFFMAN, E. (2006): Frame Analysis. Los marcos de la experiencia, Madrid, CIS-Centro de Investigaciones Sociológicas.
IGARTÚA, J.J.; MUÑIZ, C. y CHENG, L. (2005): “La inmigración en la prensa española. Aportaciones empíricas y metodológicas desde la teoría del encuadre noticioso", Migraciones, 17 , págs. 143-181.

MAULEÓN, I. y SARDÁ, J. (2014): La economía sumergida en Navarra. Working paper. Pamplona, Gobierno de Navarra.

McCOMBS, M.E. y SHAW, D.L. (1972): “The Agenda-Setting Function of Mass Media", The Public Opinion Quarterly, Vol. 36, № 2, págs. 176-187.

MUÑIZ, C. e IGARTÚA, J.J. (2004): “Encuadres noticiosos e inmigración. Un análisis de contenido de la prensa y televisión españolas", ZER Revista de Estudios de Comunicación, Vol. 9, no 16.

SANZ, L. (2003): “Análisis de redes sociales: o cómo representar las estructuras sociales subyacentes", Apuntes de Ciencia y Tecnología, 7, págs. 21-29.

VAQUERO, A.; LAGO, S. y FERNÁNDEZ, X. (2015): Economía sumergida y fraude fiscal en España. ¿Qué es lo que sabemos?, Fundación de las Cajas de Ahorros, Documento de Trabajo, nํㅜ $768 / 2015$. 